\title{
HISTOPLASMOSE EM PACIENTES IMUNODEPRIMIDOS: ESTUDO DE 18 CASOS OBSERVADOS EM UBERLÂNDIA, MG
}

\author{
Aércio S. Borges, Marcelo Simão Ferreira, Marco Túlio A. Silvestre, \\ Sérgio de A. Nishioka e Ademir Rocha
}

\begin{abstract}
Os autores descrevem dezoito casos de bistoplasmose observados em pacientes imunodeprimidos, sendo 17 com SIDA e um associado à cirrose bepática alcoólica. O diagnóstico da histoplasmose foi obtido através do isolamento do fungo em cultura de LCR, sangue e medula óssea ou por exame bistopatológico obtido por biópsia ou necropsia. A idade média dos pacientes foi de 35,8 anos, sendo 13 (72,2\%) do sexo masculino. Em geral, a doença mostrou-se disseminada com acometimento por ordem de freqüência de: pele (38,8\%), medula óssea (27,7\%), mucosa nasofaringeana (22,2\%), pulmão (22,2\%), cólon (11,1\%), SNC (5,5\%) e esôfago (5,5\%). Adenomegalia (50\%), bepatomegalia (77,7\%) e esplenomegalia (61,1\%) também foram observados com muita frequência. Hematologicamente observou-se mais comumente pancitopenia (33,3\%). Onze dos 18 pacientes receberam tratamento, sendo 9 com anfotericina $B$ e 2 com itraconazol; 8 obtiveram boa resposta clinica e todos receberam terapia de manutenção com anfotericina $B$ ou derivado triazólico. Este trabalho enfatiza a importância desta micose em pacientes imunodeprimidos, particularmente em pacientes com SIDA onde a infecção tende a acometer o sistema macrofágico-linfóide e o tegumento cutâneo.
\end{abstract}

Palavras-chaves:Histoplasmose. SIDA. Imunodepressão. Cirrose hepática.

Histoplasmose é uma micose sistêmica causada por um fungo dimórfico, o Histoplasma capsulatum, do qual duas variedades têm sido descritas: o Histoplasma capsulatum var. capsulatum e o Histoplasma capsulatum var. duboisi ${ }^{7}$. Em indivíduos imunocompetentes, este fungo, usualmente, causa infecção autolimitada ou localizada; infecção disseminada ocorre, em geral, em imunodeprimidos ${ }^{17}$. Antes da síndrome da imunodeficiência adquirida (SIDA), casos sistêmicos eram vistos predominantemente em portadores de linfomas e outras neoplasias hematológicas ou em transplantados renais ${ }^{81615}$. A partir de 1987, todos os casos de indivíduos infectados pelo vírus da imunodeficiência humana (HIV) com histoplasmose extrapulmonar foram definidos como tendo SIDA, pelo Centers for Disease Control ( CDC) ${ }^{1}$.

Em áreas endêmicas da micose, esta doença pode representar a primeira manifestação da

Centro de Ciências Biomédicas da Universidade Federal de Uberlândia, Uberlândia, MG, Brasil.

Endereço para correspondência: Prof. Marcelo S. Ferreira. Av. João Pinheiro 21/100, 38400-057 Uberlândia, MG. Fax: (034) 218-2246.

Recebido para publicação em 01/04/96.
SIDA. Nesta síndrome, como em outras doenças imunodepressoras, a histoplasmose representa, muito provavelmente, uma reativação de focos latentes de infecção pregressa ou, mais raramente, aquisição exógena, por ocasião da imunodepressão ${ }^{3913}$.

Neste trabalho, descreveremos 18 casos disseminados desta infecção, sendo 17 em pacientes com SIDA e um associado à cirrose hepática alcoóolica, ressaltando suas manifestações clínicas, laboratoriais, diagnóstico e resposta ao tratamento.

\section{MATERIAL E MÉTODOS}

Revisamos 18 prontuários de pacientes com diagnóstico de histoplasmose, sendo 17 infectados pelo HIV e um associado à cirrose hepática alcoólica, atendidos no Hospital das Clínicas da Universidade Federal Uberlândia (HC-UFU), no período de janeiro de 1985 a dezembro de 1995.

O diagnóstico de histoplasmose foi definido através do isolamento do fungo em hemoculturas, mieloculturas, cultura de líquor ou lavado brônquico e/ou pelo exame histopatológico de material obtido por biópsia ou necropsia. O diagnóstico de infecção pelo HIV foi realizado através do teste 
Borges AS, Ferreira MS, Silvestre MTA, Nishioka SA, Rocha A. Histoplasmose em pacientes imunodeprimidos: estudo de 18 casos observados em Uberlândia, MG. Revista da Sociedade Brasileira de Medicina Tropical 30:119-124, mar-abr, 1997.

imunoenzimático ELISA, em duas amostras de soro. No paciente com cirrose hepática alcóolica, o diagnóstico da hepatopatia crônica foi confirmado à necropsia.

\section{RESULTADOS}

Os achados clínicos e laboratoriais, bem como o método de diagnóstico, tratamento e evolução estão representados na Tabela 1.

Os pacientes tinham idades entre 21 e 54 anos (média de 35,8 anos), sendo 13 (72,2\%) do sexo masculino. Dezessete $(94,4 \%)$ estavam infectados pelo HIV, sendo 8 usuários de drogas injetáveis (destes, 2 tinham também comportamento homossexual e um, bissexual); 2 eram homossexuais apenas, 4 heterossexuais promíscuos e em 3 a condição predisponente para a infecção pelo HIV não foi determinada.

Os sintomas tiveram início, em média, 2 meses antes do diagnóstico. Os achados clínicos e laboratoriais mais freqüentemente encontradas foram: lesões de pele (papulares, eritemato-violáceas e/ou ulceradas) em 7/18 $(38,8 \%)$ (Figuras 1 e 2 ), infiltrado pulmonar a o Rx de tórax em 4/18 (22,2\%), lesão ulcerada de mucosa oral em 3/18 (16,6\%) (Figura 3), dor abdominal com diarréia e enterorragia em $3 / 18$ (16,6\%), comprometimento neurológico (hemiparesia) em 1/18 (5,5\%), disfagia com úlcera esofâgica, endoscopicamente diagnosticada, em 1/18 (5,5\%) e lesão de mucosa nasal em $1 / 18(5,5 \%)$. Adenomegalia generalizada foi encontrada em 9/18 (50\%), hepatomegalia em $14 / 18(77,7 \%)$ e esplenomegalia em 10/18 (61,1\%) (Figura 4). Em dois pacientes, com

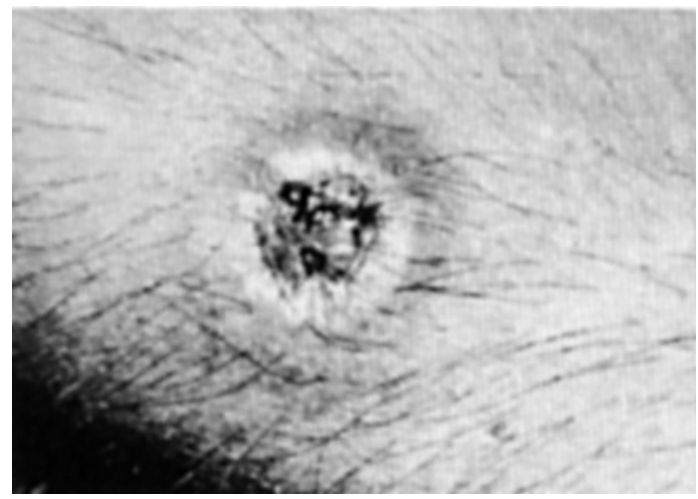

Figura 1 - Histoplasmose - Lesão cutânea ulcerada de bordas elevadas, fundo granuloso, localizada no antebraço direito (Caso 3).

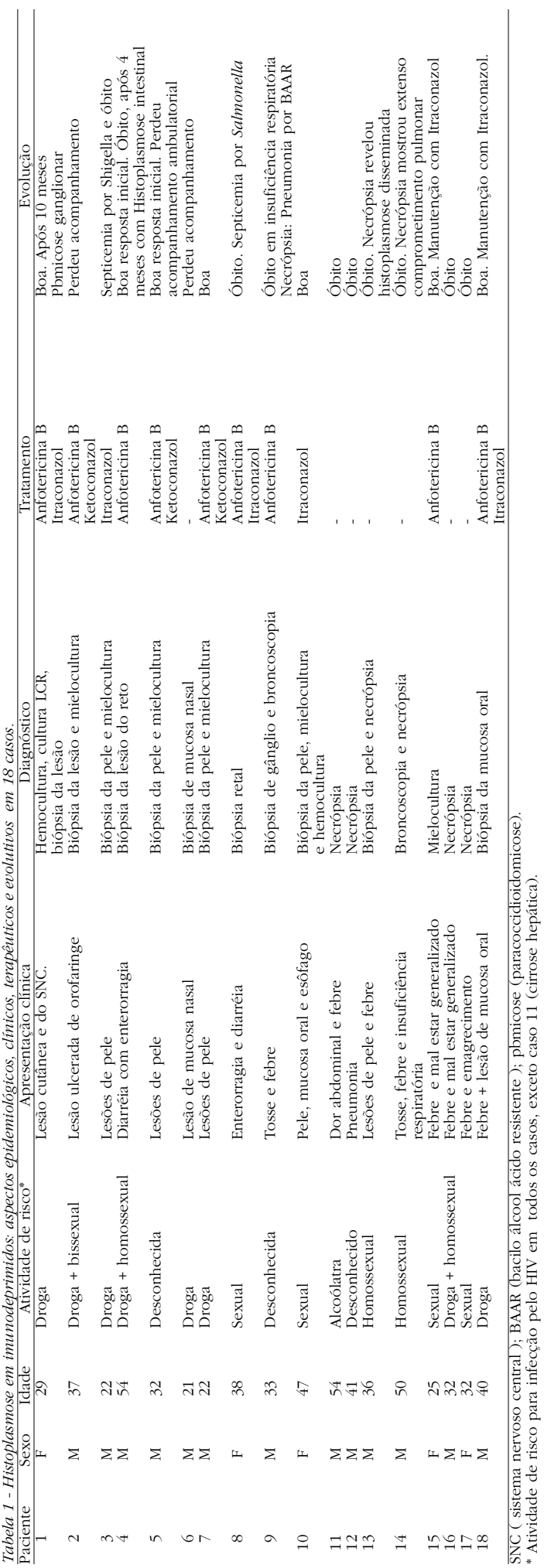


Borges AS, Ferreira MS, Silvestre MTA, Nishioka SA, Rocha A. Histoplasmose em pacientes imunodeprimidos: estudo de 18 casos observados em Uberlândia, MG. Revista da Sociedade Brasileira de Medicina Tropical 30:119-124, mar-abr, 1997.

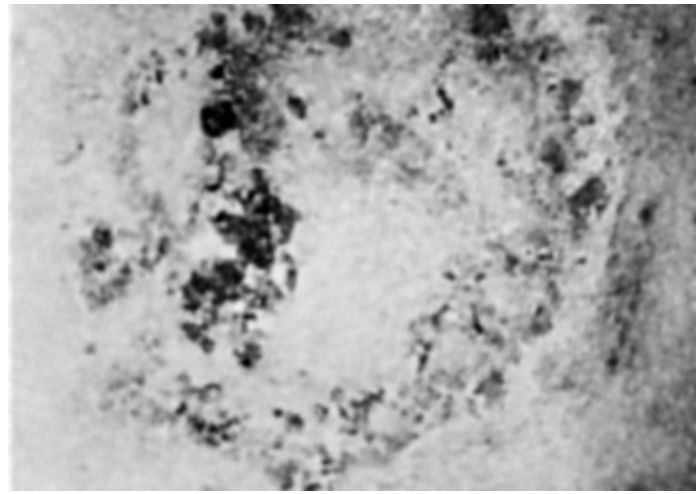

Figura 2 - Histoplasmose. Lesão cutânea extensa, eritemato-descamativa localizada na região dorsal interescapular; biópsia demonstrou o fungo (Caso 3).

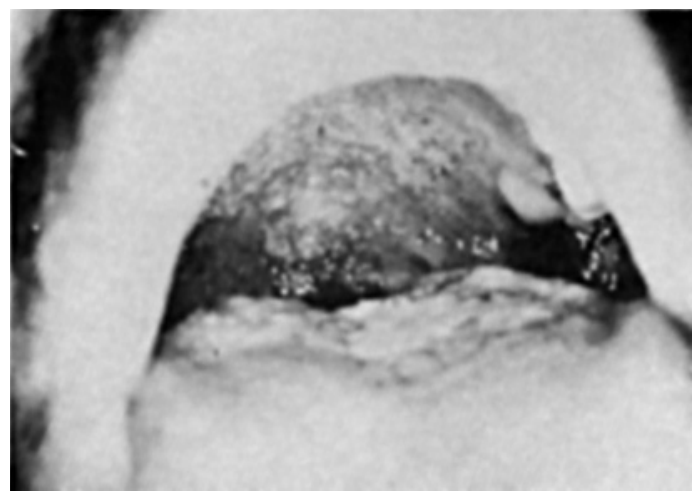

Figura 3 - Histoplasmose. Lesão da cavidade oral, infiltrativa, de aspecto granulomatoso, comprometendo palatos duro e mole, lingua e tecidos periodontais; a biópsia demonstrou o Histoplasma capsulatum (Caso 6).

diarréia, foram observados ao enema opaco e à retossigmoidoscopia, lesões tumorais, cujo exame histopatológico revelou a presença do fungo (Figura 5).

Dos 17 casos avaliados hematologicamente, $16(94,1 \%)$ apresentavam-se com anemia, 6 $(33,3 \%)$ com pancitopenia (Leucócitos $<3500 / \mathrm{mm}^{3}, \mathrm{Hb} \leq 10 \mathrm{mg} \%$ e plaquetas $\left.<100000 / \mathrm{mm}^{3}\right), 6(33,3 \%)$ apresentaram anemia e leucopenia, e 2 (11,1\%), anemia e plaquetopenia.

Com relação ao diagnóstico, o fungo foi encontrado ao exame histopatológico (biópsia de pele e/ou mucosa) em $100 \%$ dos casos com comprometimento mucocutâneo, em

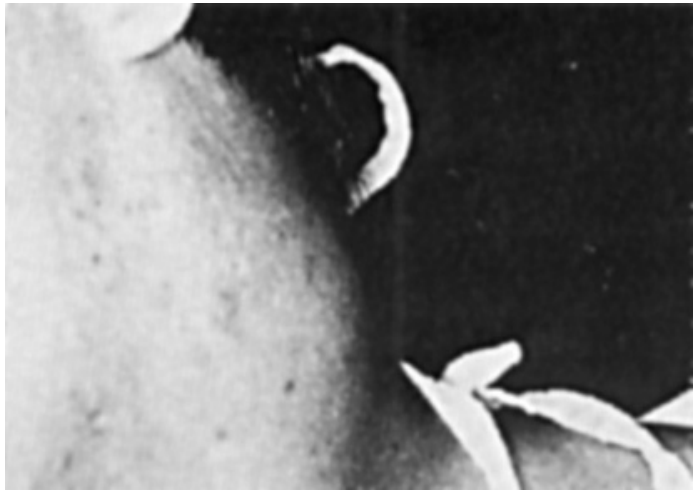

Figura 4 - Histoplasmose. Adenomegalia cervical coalescida; punçao aspirativa demonstrou o agente (Caso 9).

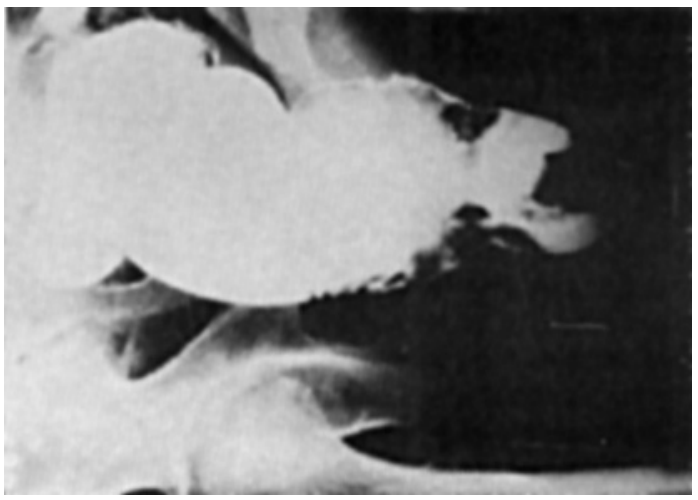

Figura 5 - Histoplasmose - Enema opaco demonstrando lesão tumoral no reto, confundida com adenocarcinoma, cuja biópsia demonstrou a presença do Histoplasma capsulatum (Caso 4).

hemoculturas em 3/18 casos (16,6\%) e na mielocultura em 5/18 (27,7\%). No caso 1, com comprometimento do sistema nervoso central (SNC), o fungo foi isolado do líquor e sangue através de cultura e identificado no exame histopatológico da pele; o paciente, após remissão da doença, abandonou o tratamento e, no retorno, 10 meses após, apresentava-se com adenomegalia axilar, hepatoesplenomegalia e febre. A biópsia do gânglio revelou a presença de paracoccidioidomicose.

Em seis casos, o diagnóstico só foi realizado post-mortem, através da necropsia (5 com SIDA e um com cirrose hepática alcoólica), sendo o fungo encontrado em 
Borges AS, Ferreira MS, Silvestre MTA, Nishioka SA, Rocha A. Histoplasmose em pacientes imunodeprimidos: estudo de 18 casos observados em Uberlândia, MG. Revista da Sociedade Brasileira de Medicina Tropical 30:119-124, mar-abr, 1997.

fígado (Figura 6), baço, gânglios (Figura 7) e pulmões em 6/6 (100\%); nas supra-renais e rins em 2/6 (33,3\%); no pâncreas e miocárdio em $1 / 6(16,6 \%)$.

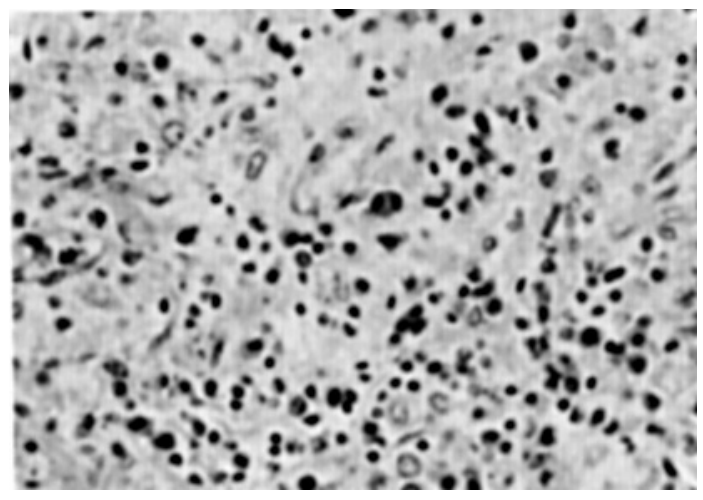

Figura 6 - Histoplasmose. Corte bistológico do fígado retirado à autópsia de doente com cirrose hepática e histoplasmose disseminada. Notar macrófagos com fungos no centro da foto - $400 X$ (Caso 11).

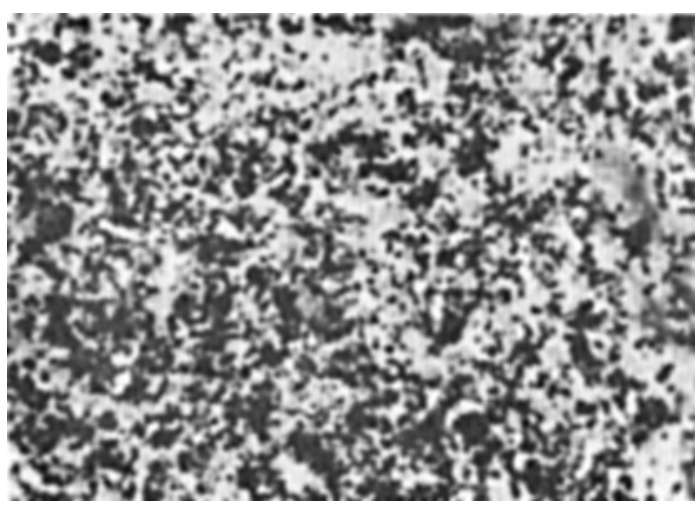

Figura 7 - Histoplasmose. Corte histológico de linfonodo, corado pelo Grocott, observando enorme quantidade de formas leveduriformes do Histoplasma capsulatum (1000X - imersão) (Caso 9).

Quanto ao tratamento, 9 receberam inicialmente anfotericina $\mathrm{B}$ (doses totais de $400 \mathrm{mg}$ a $2 \mathrm{~g}$ ) e 2 , itraconazol $(400 \mathrm{mg} / \mathrm{dia})$. Em 1 paciente (no 6 da Tabela 1) o tratamento não foi instituído por abandono do acompanhamento. Dos 11 casos tratados, 8 tiveram boa resposta inicial, sendo 2 com itraconazol e $6 \mathrm{com}$ anfotericina B. Todos receberam terapia de manutenção após remissão, com anfotericina $\mathrm{B}, 1 \mathrm{mg} / \mathrm{kg} /$ dose, uma vez por semana ou com ketoconazol ou itraconazol diariamente (Tabela 1). Três pacientes evoluíram para o óbito durante a hospitalização, sendo um com sepse por Shigella, um por Salmonella e um com insuficiência respiratória causada pelo próprio fungo. Até dezembro de 1995 apenas 2 pacientes permaneciam vivos, em tratamento de manutenção com itraconazol (casos 15 e 18 da Tabela 1).

\section{DISCUSSÃO}

Histoplasmose generalizada comumente ocorre por disseminação hematogênica do fungo a partir de um foco pulmonar primário. Esta forma de apresentação, entretanto, era rara até o surgimento da SIDA, ocorrendo apenas em extremos de idade e em indivíduos imunodeprimidos por doenças neoplásicas ou pelo uso de terapia imunossupressora (transplantados, colagenoses, etc.).

A associação de histoplasmose e SIDA é mais frequentemente encontrada onde a micose é endêmica, com prevalência variando entre 2 e 30\%, de acordo com a região estudada'. Na grande maioria dos casos, a doença representa reativação de focos latentes da infecção, embora haja casos bem demonstrados de infecção primária com disseminação ${ }^{3914}$.

$\mathrm{Na}$ presente casuística, em 22,2\% dos casos ocorridos em pacientes infectados pelo HIV, a histoplasmose foi a primeira manifestação da doença, tendo sido, portanto, a infecção que definiu o diagnóstico de SIDA.

O $H$. capsulatum causa doença disseminada em mais de $95 \%$ dos casos de SIDA ${ }^{15}$. A doença pode apresentar-se com início agudo, fulminante, semelhante a uma síndrome séptica por bactérias Gram-negativas. Entretanto, a apresentação mais comum é de evolução subaguda, que se arrasta por semanas, com sintomas respiratórios e acometimento do sistema macrofágico-linfóide (adenomegalia e hepatoesplenomegalia ${ }^{15}$. A maioria dos nossos casos mostrou este tipo de evolução. Ressaltamos a freqüência com que as lesões cutâneas foram vistas em nossos pacientes (38,8\%); o acometimento da pele na histoplasmose disseminada em pacientes com SIDA, em geral, ocorre em 10 a $25 \%$ dos casos e as lesões 
Borges AS, Ferreira MS, Silvestre MTA, Nishioka SA, Rocha A. Histoplasmose em pacientes imunodeprimidos: estudo de 18 casos observados em Uberlândia, MG. Revista da Sociedade Brasileira de Medicina Tropical 30:119-124, mar-abr, 1997.

comumente são pleomórficas, fato também observado nesta casuística ${ }^{411} 1315$.

O comprometimento do trato gastrointestinal (TGI) pelo $H$. capsulatum é raro e parece resultar também de disseminação hematogênica a partir de foco pulmonar. Entretanto, o TGI pode servir de porta de entrada para esta infecção, particularmente em lactentes ${ }^{6}{ }^{10}$. Nesse estudo, em 3 casos $(16,6 \%)$, a manifestação inicial foi o comprometimento do TGI, com diarréia sanguinolenta e dor abdominal. Destes (2 com SIDA e um com hepatopatia crônica), o fungo foi evidenciado, pelo exame histopatológico, em material obtido por biópsia retal, através de retossigmoidoscopia em 2 e no terceiro, através de necropsia.

O SNC pode estar acometido isoladamente, ou seja, sem evidências clínicas da infecção em outros sítios ou, mais comumente, como foco de disseminação hematogênica a partir de outros órgãos. A prevalência varia também com a área geográfica considerada, chegando a 29 \% em uma série de casos fatais de histoplasmose disseminada, estudados por necropsia. As formas de apresentação clínica no SNC relatados na literatura são: histoplasmoma, que se comporta como uma lesão ocupando espaço no encéfalo; meningite crônica, muito similar àquela vista na tuberculose, podendo ocorrer isoladamente ou como foco de disseminação; êmbolos sépticos associados a endocardite e, mais raramente, encefalite ${ }^{21416}$.

Encontramos, em nosso material, um caso de histoplasmose do SNC (caso 1), manifestandose com sinais clínicos de envolvimento focal, cuja tomografia computadorizada de crânio mostrou lesão expansiva, hipodensa, com reforço após injeção de contraste, similar aquelas vistas na reativação de toxoplasmose ou tripanossomíase cruzi no SNC. A análise do líquor revelou - se normal e o fungo foi isolado deste material através de cultura.

Face à gravidade da infecção pelo $H$. capsulatum em imunodeprimidos, preconizou - se, como terapêutica inicial de supressão, a anfotericina B (doses totais de 2 a $3 \mathrm{~g}$ ) e, para aqueles que alcançarem remissão clínica e micológica, terapêutica de manutenção preferentemente com itraconazol ou com infusões semanais de anfotericina B $(50 \mathrm{mg} / \mathrm{dose})^{4}{ }^{15}$. Recentemente, alguns autores têm ressaltado a eficácia do itraconazol oral (400mg/dia ) como terapêutica inicial para esta micose $^{15}$, fato por nós comprovado em alguns de nossos casos (pacientes 3 e 10 da Tabela 1).

Em conclusão, os autores enfatizam a importância da histoplasmose em pacientes imunodeprimidos, particularmente em pacientes com SIDA, cuja infecção tende a acometer principalmente o sistema macrofágico-linfóide e a pele. A lembrança deste diagnóstico deve recair, principalmente, sobre indivíduos que habitam ou habitavam áreas sabidamente endêmicas. A inclusão rotineira dos meios de cultura para fungos em amostras de sangue, exsudatos cutâneos, secreções respiratórias, líquor e outros humores na prática clínica diária poderá aumentar a frequência do diagnóstico desta micose em nosso meio.

\section{SUMMARY}

The authors describe eighteen cases of histoplasmosis in immunodepressed patients, 17 with the acquired immunodeficiency syndrome (AIDS) and one patient with hepatic cirrbosis. The diagnosis of histoplasmosis was made by isolation of Histoplasma capsulatum from cerebrospinal fluid (CSF), blood and bone marrow or by histopathologic (biopsy or post mortem) examination. The mean age of the patients was 35.8 years; 13 patients were male $(72.2 \%)$. The disease was disseminated, with the following distribution:skin (38.8\%), bone marrow (27.7\%), nasopharyngeal mucosa (22.2\%), lungs (22.2\%), cólon (11.1\%), central nervous system (5.5\%) and esophagus (5.5\%). Adenomegaly (50\%), hepatomegaly (77.7\%) and splenomegaly (61.1\%) were frequently seen. The most common hematologic abnormality was pancytopenia (33.3\%) of the patients. Eleven patients were treated, 9 with amphotericin B and 2 with itraconazole. Eight had good clinical improvement and all of them were given amphotericin $B$ or a triazolic as maintenance therapy. This study emphasize the importance of this mycosis in imunodepressed patients, specially AIDS patients, in whom the infection tends to invade the macrophagic-lymphoid system and preferentially the cutaneous tegument.

Key-words: Histoplasmosis. AIDS. Immunosupression. Hepatic cirrhosis.

\section{AGRADECIMENTOS}

Agradecemos ao Dr. Leandro P. Moura por seu auxílio na execução técnica do trabalho. 
Borges AS, Ferreira MS, Silvestre MTA, Nishioka SA, Rocha A. Histoplasmose em pacientes imunodeprimidos: estudo de 18 casos observados em Uberlândia, MG. Revista da Sociedade Brasileira de Medicina Tropical 30:119-124, mar-abr, 1997.

\section{REFERÊNCIAS BIBLIOGRÁFICAS}

1. Centers for Disease Control. Revision of the CDC surveillance case definition of acquired immunodeficiency syndrome. The Journal of the American Medical Association 258:1143-1154, 1987.

2. Cooper RA, Goldstein E. Histoplasmosis of the central nervous system: Report of two cases and review of literature. The American Journal of Medicine 35:45-57, 1963.

3. Dickinson DJ, Durry E. Epidemiology of histoplasmosis among AIDS patients during a community - wide outbreak in 1988-90, Marion County (Indianapolis), Indiana. Program and abstracts of 31st Interscience Conference an Antimicrobiol Agents and Chemotherapy, Anaheim, American Society for Microbiology, 1992.

4. Eidbo J, Sanchez RL, Tschen JA and Ellner KM. Cutaneous manifestations of histoplasmosis in the AIDS. The American Journal of Surgical Pathology 17:110-116, 1993.

5. Goodwin RA, Shapiro JL, Thurman GH, Thurman SS, Desprez RM. Disseminated histoplasmosis: clinical and pathologic correlations. Medicine (Baltimore) 59:1-33, 1980.

6. GrahansBD, Mc Kinsey DS, Duks MR and Smith DL. Colonic histoplasmosis in Acquired Immunodeficiency Syndrome. Diseases of Cólon and Rectum 34:185-190, 1991.

7. Keath EJ, Kabayashi GS, Medoff G. Typing of Histoplasma capsulatum by restric ion fragment length polymorphisms in a nuclear gene. Journal of Clinical Microbiology 30:2104-2107, 1992.

8. Machado AA, Coelho ICBC, Roselino AMF, Trad ES, Figueiredo JF, Martinez R, Costa JC. Histoplasmosis in individuals with Acquired Immune Syndrome (AIDS): Report of six cases with cutaneous mucosal involvement. Mycopathologia 115:1318,1991
9. Mc Kinsey DS, Brewer J, Niehart R. Risk factors for histoplasmosis in AIDS:Results from on ongoing cohort study (Abstract 60). In: Abstracts of the 1992 IDSA Meeting, Infectious Diseases Society of America, Anaheim p. 22, 1992.

10. Miller DP, Everett ED. Gastrointestinal histoplasmosis. Journal of Clinical Gastroenterology 1:233-236, 1979.

11. Negroni R, Robles AM, Arechavala A, Taborda A. Histoplasmosis diseminada en pacientes con SIDA, su evolución y tratamiento. Revista Argentina de Micologia 14:5-12, 1991.

12. Neubaer MA and Bodensteiner DC. Disseminated histoplasmosis in patients with AIDS. Southern Medical Journal 85:1166-1170, 1992.

13. Rocha, MM, Severo LC. Histoplasmose disseminada em pacientes com Síndrome de Imunodeficiência Adquirida. Revista do Instituto de Medicina Tropical de São Paulo 36:167-170, 1994.

14. Smith JW, Utz JP. Progressive disseminated histoplasmosis; A prospective study of 26 patients. Annals of Internal Medicine 76:557, 1972.

15. Wheat J. Endemic mycoses in AIDS: a clinical review. Clinical Microbiology Reviews 8:146-159, 1995.

16. Wheat LJ, Botterger BE, Sathapatayavongs B. Histoplasma capsulatum infections of the central nervous system. A clinical review. Medicine 69:244-260, 1990.

17. Wheat LJ, Stringfield PAC, Baker RL, Curfiman MF, Eads ME, Israel KS, Norris SA, Webb DH, Zeckel ML. Disseminated histoplasmosis in the Acquired Immune Deficiency Syndrome: clinical findings, diagnosis and treatment, and review of the literature. Medicine 69:361-374, 1990. 